The following scientific article was officially published in the journal Spine, published by Lippincott Williams \& Wilkins (Wolters Kluwer). This is a non-final version of an article published in final form in:

Seoud, L., Dansereau, J., Labelle, H. and Cheriet, F. "Multilevel Analysis of Trunk Surface Measurements for Noninvasive Assessment of Scoliosis Deformities.” Spine, Vol. 37, No. 17, (2012), pp.E1045-E1053.

The final publication is available via the link: http://dx.doi.org/10.1097/BRS.0b013e3182575938

The manuscript, as accepted by the publisher, is reproduced in the following pages.

Copyright (C) 2012 Lippincott Williams \& Wilkins. Unauthorized reproduction of this article is prohibited. 


\section{MULTILEVEL ANALYSIS OF TRUNK SURFACE MEASUREMENTS FOR NON- INVASIVE ASSESSMENT OF SCOLIOSIS DEFORMITIES}

Lama Séoud $^{1,2}$, Jean Dansereau ${ }^{1,2}$, Ph.D., Hubert Labelle ${ }^{2}$, M.D., and Farida Cheriet ${ }^{1,2}$, Ph.D.

(1) École Polytechnique de Montréal, Québec, Canada

(2) Sainte Justine Hospital Research Center, Montréal, Québec, Canada

This work was supported by the Natural Sciences and Engineering Research Council of Canada, the Fonds Québecois de Recherche sur la Nature et les Technologies, and MENTOR, a strategic training program of the Canadian Institutes of Health Research.

Corresponding author and address for reprints:

Lama Séoud

École Polytechnique de Montréal

Department of Biomedical Engineering

P.O. Box 6079

Montréal, Québec

Canada H3C 3A7

Tel 1: 514-340-4711 (Ext: 5050)

Tel 2: 514-802-5337

Fax: 514-340-4658

E-mail: lama.seoud@polymtl.ca 
Study design: Reliability study.

Objectives: To assess between-acquisition reliability of new multi-levels trunk cross-sections measurements, in order to define what is a real change when comparing two trunk surface acquisitions of a same patient, before and after surgery or throughout the clinical monitoring.

Summary of background data: Several cross-sectional surface measurements have been proposed in the literature for non-invasive assessment of trunk deformity in patients with adolescent idiopathic scoliosis (AIS). However, only the maximum values along the trunk are evaluated and used for monitoring progression and assessing treatment outcome.

Methods: Back surface rotation (BSR), trunk rotation (TR) and coronal and sagittal trunk deviation are computed on 300 cross-sections of the trunk. Each set of 300 measures is represented as a single functional data using a set of basis functions. To evaluate betweenacquisition variability at all trunk levels, a test-retest reliability study is conducted on 35 AIS patients. A functional correlation analysis is also carried out to evaluate any redundancy between the measurements.

Results: Each set of 300 measures was successfully described using only 10 basis functions. The test-retest reliability of the functional measurements is good to very good all over the trunk, except above the shoulders levels. The typical errors of measurement are between $1.20^{\circ}$ and $2.2^{\circ}$ for the rotational measures and between 2 to $6 \mathrm{~mm}$ for deviation measures. There is a very strong correlation between BSR and TR all over the trunk, a moderate correlation between coronal trunk deviation and both BSR and TR and no correlation between sagittal trunk deviation and any other measurement.

Conclusions: This novel representation of trunk surface measurements allows for a global assessment of trunk surface deformity. Multilevel trunk measurements provide a broader perspective of the trunk deformity and allow a reliable multi-level monitoring during clinical follow-up of patients with AIS and a reliable assessment of the esthetic outcome after surgery. 
KeY WoRDS: Scoliosis, multi-level trunk surface measurement, functional data analysis, reliability, correlation.

\section{KEY POINTS:}

1) Trunk cross-sections measurements are reliable all along the trunk, except at the shoulders level.

2) The maximum value of a measurement along the trunk overlooks the extent of the hump above and below the apex of the curve.

3) Functional trunk deviation and axial rotations provide complementary information about trunk deformity.

4) With our non-invasive multi-level trunk surface analysis, it is possible to detect a significant progression of the spinal curvature and to document the esthetic outcome of a surgery.

5) Although the range of the measurements is not the same, radiographic and trunk surface measurements profiles of a typical patient show similar variations.

\section{Mini ABStRACt / PrÉCIS:}

This study assesses the reliability of multi-levels trunk cross-sections measurements and defines the difference needed between trunk surface acquisitions to detect a real progression and/or surgical correction of trunk surface deformity at all trunk levels. 


\section{INTRODUCTION}

The spinal deformity associated to adolescent idiopathic scoliosis (AIS) affects the general appearance of the trunk in a more or less significant way, depending on its type and severity. Assessing trunk surface deformities is valuable in clinic since it documents an important aspect in patients' eyes [1-2].

This motivation has led to the development of 3D scanning systems [3-9] for the assessment of trunk deformities. All these systems provide a 3D reconstruction of the back or the entire torso with different resolutions depending on the systems. As opposed to radiographic acquisition, these systems use only unharmful visible light. Although these devices are valuable and promising, the major obstacle to their common use in clinic is currently the unclear definition of trunk metrics.

Numerous topographic measurements have been proposed in the literature starting with angles similar to the Cobb angle, computed from the spinous process line [10,11], the back valley line [12] or the trunk cross-sections centroid line [13]. Easier to correlate with the Cobb angle, they document only the deformity in the coronal plane. Several scores and index were also suggested [14-17], most of which suffer from poor inter- and intra-operator reliability associated with the manual identification of numerous landmarks on the back surface. Another promising approach consists in computing local measurements on trunk cross-sections, such as the back surface rotation (BSR) $[12,13,18]$ similar to the angle of trunk inclination (ATI) $[19]$ and the trunk rotation $[13,18]$.

The quantitative analysis of trunk deformities using trunk surface acquisition is valuable in clinic. First, it provides a radiation-free alternative for a more frequent follow-up of AIS patient. Second, it can be used, in addition to radiographs, to assess the esthetic outcome of a spinal surgery. Nevertheless, when comparing two trunk surface acquisitions of a same patient, before and 
after surgery or throughout the follow-up, it is important to distinguish between a real change and a change associated to a difference in patient's posture between acquisitions.

Previous work in our group evaluated the reliability of trunk surface cross-sectional measurements [18] but taking into consideration only the maximum values along the trunk. In fact, in the trunk cross sectional approach, the entire multi-level measurement profile is usually reduced to a single measurement, as the maximum value along the trunk, in order to facilitate analysis using classical statistical methods.

Maximum values of trunk cross-sectional measurements provide certainly an idea of the severity of trunk deformations; however, it overlooks the extent and the location of the deformations. During AIS follow up, the maximal amplitude of trunk deformity may remain the same from one trunk surface acquisition to another; however, changes may occur elsewhere along the trunk. The hypothesis of this work is thus the following: taking into consideration the whole measurements profile can provide more and novel information for clinicians when analyzing trunk surface deformities associated with scoliosis.

Multi-segmental analysis of the trunk deformity has already been proposed in the scoliosis literature. It uses the scoliometer to measure the angle of trunk inclination (equivalent to our BSR) at 10 levels between $\mathrm{C} 7$ and S1 [20, 21]. This method has been highly recommended for the assessment of scoliosis spinal surgery outcome [22]. Unfortunately it has several limitations, starting with controversial evidence regarding the scoliometer reliability and validity [23, 24]. Moreover, the method requires the patient to bend forward. This posture alters the magnitude of trunk deformity, is hard to standardize and multi-level scoliometer measurement is timeconsuming. The use of optical systems overcomes those limitations.

Since we are interested in how the measurements fluctuate at each trunk level, each set of values along the trunk constitutes an observation or a datum in itself. This approach is known as functional data analysis (FDA) [25]. Functional data analysis is an emerging topic in statistics. It has been successfully used in the medical field for the classification of temporal gene 
expression data $[26,27]$ and for the analysis of normal and pathological kinematic gait data [2830].

Consequently, the first objective of this paper is to propose new multi-levels trunk surface measurements for the assessment of trunk deformities associated to AIS. Then, in order to assess what is a real change in the measurements between different trunk surface acquisitions of a patient, the second objective of this study is to evaluate the reliability of the proposed measurements, not only for the maximum values along the trunk but at all trunk levels. And finally, because clinicians gold standard for the radiographic assessment of scoliosis is resumed into a single measurement, the Cobb angle, we are interested in reducing as much as possible the number of measurements for the topographic assessment. Thus, our third objective is to study the correlations between our measurements in order to propose a compact set of measurements.

\section{MATERIALS AND MethodS}

\section{Clinical dataset}

We used the data of 32 AIS patients who attended the Sainte Justine Hospital Research Center (SJHRC) for their routine pre-operative visit. All subjects were enrolled on a voluntary basis and an informed consent was obtained for each patient as approved by the Ethical Committee of the institution. There were 26 girls and 6 boys. The main radiological and demographic characteristics of the cohort are summarized in Table 1.

\section{TRUNK ACQUISITION PROTOCOL}

Currently at SJHRC, the trunk surface is acquired using an active vision system, the Inspeck system (Creaform®), Québec, Canada) as described in [9]. The acquisition time is of 4 to 5 seconds. The accuracy of the reconstruction is of $1.1 \pm 0.9 \mathrm{~mm}$ over the entire trunk surface [9]. 
In order to study the reliability of trunk measurements, each patient's trunk was acquired twice during the same visit. The patients were asked to stand still in the upright position with the arms slightly abducted in order not to obstruct the lateral scanners' fields of view. Between acquisitions, patients relaxed at least half a minute before repositioning. The posture was explained and demonstrated before the acquisition series.

Prior to the first acquisition, a nurse located 4 anatomic landmarks on the trunk by palpation and places markers over them. These landmarks are the left and right anterior-superior iliac spines (ASIS), the posterior-superior iliac spines' midpoint (MPSIS) and C7 vertebral prominence (VP).

\section{PATIENT-SPECIFIC REFERENCE FRAME}

The trunk surface 3D reconstruction is transposed into a patient-specific 3D reference frame, equivalent to the SRS whole body coordinate system [31]. It is defined such that: the origin is the MPSIS, the Y-axis is the vertical up; the X-axis is the horizontal parallel to the ASIS line, oriented toward the right of the patient; the Z-axis is obtained by cross-product and oriented toward the patient's back.

For ends of comparison, another patient-specific frame $\left(X^{\prime}, Y^{\prime}, Z^{\prime}\right)$ is considered. It is defined such as the vertical axis $Y^{\prime}$ passes by the VP and the MPSIS, $X^{\prime}$ is the projection of the ASIS line onto the plane $Y^{\prime}=0$, and $Z^{\prime}$ is the cross-product of $X^{\prime}$ and $Y^{\prime}$. This coordinate system is equivalent to the SRS spinal coordinate system [31]. It is believed that this coordinate system compensate for postural changes in the upper trunk, since the vertical axis is defined by the VP.

\section{TRUNK FEATURES EXTRACTION}

300 horizontal cross-sections corresponding to the different vertebral levels between L5/S1 and $\mathrm{C} 7 / \mathrm{T} 1$, equally spaced along the $\mathrm{Y}$-axis, are automatically extracted starting from the MPSIS and going up to the VP. The distance between consecutive cross-sections depends on the subject's height. In average, on our cohort, the distance is $1.3 \pm 0.1 \mathrm{~mm}$. At each trunk level, an ellipse is fitted to the cross-section points using a direct least-squares fitting method [32]. A 
section-specific reference frame $(G, U, V)$ is then defined The ellipse's center is considered as the section's center $\mathrm{G}$. The $\mathrm{U}$ and $\mathrm{V}$ axis correspond respectively to the ellipse's major and minor axis. In this reference frame, the dual tangent (DT) to the back side of the section is defined as the tangent to the left and right back portions of the section. In some sections a dualtangent does not exist. In these cases, the DT is the line tangent to the back section at the intersection with the V-axis.

On each cross-section, 4 measurements are automatically computed:

- The back surface rotation (BSR) is the axial angle between the section's DT and the frontal plane.

- The trunk axial rotation (TR) is the axial angle between the section's U-axis and the frontal plane.

- The X-coordinate of the section's center $G\left(X_{G}\right)$ corresponding to the section's deviation in the coronal plane.

- The Z-coordinate of the section's center $G\left(Z_{G}\right)$ corresponding to the section's deviation in the sagittal plane.

Viewed from bottom, the section's BSR and TR are positive when the DT and U-axis are rotated counter-clockwise relative to the frontal plane.

When computed at all trunk levels, the resulting 4 measurements profiles present some noise; it is mainly associated to the accuracy of the trunk surface acquisition system (accuracy of 1.4 $\mathrm{mm}$ ) and the sampling used to reduce the size of the mesh (depending on the trunk height, a mesh is composed of 40000 to 60000 points after sampling).

\section{FUNCTIONAL DATA ANALYSIS}

Instead of analyzing a trunk surface measurement as a vector of 300 noisy values computed discretely along the trunk, we considered a functional representation of the measurement. It 
consists in fitting a smooth function $f(t)$, where $t$ indicates trunk levels between the MPSIS and the VP, to the 300 raw values. The function $f(t)$ is a linear combination of $K$ elementary functions $\left\{\phi_{k}\right\}$, weighted by $K$ coefficients $\left\{a_{k}\right\}$ :

$$
f(t)=\sum_{k=1}^{K} a_{k} \phi_{k}(t)
$$

$\left\{\phi_{k}\right\}$ is a set of linearly independent functions, called basis functions that, in a linear combination, can represent all the functions of the space in which our functional measurements are included. Based on existing guidelines [33], we used cubic B-splines as our basis functions. The number $K$ of basis functions controls the smoothness of $f(t)$. The more basis functions, the better the fit to the raw values but the higher the risk of fitting noise that we would like to ignore. On the other hand, if $K$ is too small, we may miss important aspects of the function. To determine its optimal value, we let $K$ vary between 4 (minimum number of basis when considering cubic splines) and 300 (maximum imposed by the number of raw values) and for each value, we recorded the root mean squared error (RMSE) between $f(t)$ and the raw measurements values. We refer the interested readers to [34] for a more detailed technical explanation of the functional representation.

At this point, we have 4 smooth functional measurements. The same basis functions $\left\{\phi_{k}\right\}$ are used for all patients, only the coefficients $\left\{a_{k}\right\}$ are patient-specific. It follows that the functional representation is a way of reducing the dimensionality of the data. In fact, instead of describing 
a patient's measurement profile using 300 values along the trunk, we can now represent it using only a set of $K$ coefficients, the $\left\{a_{k}\right\}$.

\section{RELIABILITY STUDY}

To assess the reliability of the proposed measurements at all trunk levels, we compared the functional measurements computed all along the trunk length on 2 successive acquisitions with repositioning for each of the 32 patients in our cohort. A two-way fixed effects analysis of variance (ANOVA) with subject and acquisition as factors is performed, independently for each of the 4 measurements. We used the intra-class correlation coefficient $\left(\operatorname{ICC}_{3,1}\right)$ [35] and the typical error of measurement (TEM) [36, 37] as measures of reliability [36]. They are also represented as functions along the trunk length.

Using the between-acquisition TEM, we can define for each of the 4 measurements, the minimal difference (MD) between two acquisitions needed for the difference to be considered real. The MD is calculated as follows [37]:

$$
M D=T E M \times \sqrt{2} \times 1.96
$$

Consequently, for all subjects whose differences between acquisitions are at least greater or equal to the MD, 95\% would reflect real differences.

\section{CORRELATION ANALYSIS}

In a functional scheme, we can calculate the correlation at every two points along the functions. Thus, the correlation of two functional data returns a $2 \mathrm{D}$ map [33] that plots the Pearson correlation coefficient $(\mathrm{R})$ at every two trunk levels combination. In order to assess the correlations between the 4 functional measurements, each two measurements were paired and a functional correlation analysis was conducted for each pair.

\section{RESULTS}


A graphical user interface (Figure 1) was built using the MATLAB® software for the visualization of the trunk 3D reconstruction, the trunk cross-sections and the 4 functional measurements. Once the $3 \mathrm{D}$ model is reconstructed, the overall processing time including the landmarks manual identification is less than 60 seconds.

\section{MEASUREMENTS SMOOTHING}

A way to fix the smoothness of a function is to limit the number of its basis functions. Figure 2 clearly shows that the more basis functions are used to represent the functional measurements, the better the fit to the original data. However, we want to smooth the measurements profiles and remove the noise in the measurements. In our experiment, with $K=10$ basis functions, the residual errors between the raw and smoothed data are inferior to the upper limit TEM reported by Pazos et al [17] for the maximum values of $B S R, T R$ and $X_{G}$. Moreover, $X_{G}$ and $Z_{G}$ residual errors are below the accuracy of the acquisition system $(1.4 \mathrm{~mm})$. Thus, for the remainder of the study, we used 10 basis functions for all the measurements.

\section{MeAsurements ReLIABILITY}

Between acquisition ICCs and TEMs are presented in Figure 3. We considered that the reliability was very good for ICC above 0.95 , good between 0.85 and 0.94 and moderate between 0.60 and 0.85 .

For BSR and TR, the test-retest reliability is good to very good at almost all trunk levels, except for the most cephalic part of the trunk corresponding to the shoulders levels. For trunk levels below the shoulders, the test-retest TEM varies between $1.20^{\circ}$ and $2.05^{\circ}$ for $\mathrm{BSR}$ and between $1.40^{\circ}$ and $2.20^{\circ}$ for TR. This is substantially in agreement with the results of our previous reliability study [17] that reported typical error of $1.75^{\circ}$ and $1.04^{\circ}$ respectively for BSR and TR maximum values. 
For $\mathrm{X}_{\mathrm{G}}$ and $\mathrm{Z}_{\mathrm{G}}$, the test-retest reliability is nearly very good at all trunk levels. It is interesting to note however that the corresponding TEMs increase almost linearly while going upward along the trunk, toward the VP. Even though the measurements are computed in a patient-specific coordinate system, this does not completely compensate for postural changes. The test-retest typical error on VP's 3D coordinates is $5.16 \mathrm{~mm}, 3.76 \mathrm{~mm}$ and $6.97 \mathrm{~mm}$ respectively along $\mathrm{X}, \mathrm{Y}$ and $Z$ axis. In fact, our reference frame is only defined with respect to the pelvic region. The orientation of the vertical axis is extrinsic to the patient's trunk.

In the ( $\left.X^{\prime}, Y^{\prime}, Z^{\prime}\right)$ coordinate system, the TEMs for BSR and TR are almost unchanged (Figure 4) while the errors on the deviation measures seems to be quiet constant along the trunk levels, except for an increase in $X_{G}$ in the higher $1 / 3$ part of trunk. The errors vary slightly around 2.7 $\mathrm{mm}$ all along the trunk for $Z_{G}$ and between $3.1 \mathrm{~mm}$ for $X_{G}$ below mid-thoracic level and increases rapidly for higher thoracic levels.

\section{CORRELATIONS BETWEEN MEASUREMENTS}

The highest correlation $(R>0.9)$ is found between $f_{B S R}$ and $f_{T R}$, more specifically along the ascending diagonal of the correlation map, which means that the measurements made on the exact same cross-section are strongly correlated. This was expected since these measurements are computed in the same plane and both quantify the axial rotational component of the trunk deformity.

The correlation maps between $f_{X G}$ and each of $f_{B S R}$ and $f_{T R}$ present a more horizontal distribution. It seems that the $f_{B S R}$ profile is well correlated $(R=0.80)$ to a single trunk level of $f_{X G}$ (approximately around $T 12 / L 1$ level), and that $f_{T R}$ is moderately correlated $(R=0.75)$ to the same level's $f_{X G}$. In both cases, there is no strong correlation between paired trunk levels. No relevant correlation was noted between $\mathrm{f}_{\mathrm{ZG}}$ and any other measurement. 
It is interesting to note that, when transposed to the $\left(X^{\prime}, Y^{\prime}, Z^{\prime}\right)$ reference frame, the trunk deviation in the frontal plan $f_{X G}$ is more correlated to the rotational measures $f_{B S R}$ and $f_{T R}$ at each trunk level.

\section{NON INVASIVE FOLLOW-UP: CASE STUDY}

We used the reliability study results to compare two trunk surfaces of a 16 years old male AIS patient followed up at our scoliosis clinic (Figure 5). The surfaces are acquired 8 months apart. To determine if there is a real and significant change between acquisitions, we used the MD interval computed from equation (2). Figure 6 illustrates the functional $B S R, X_{G}$ and $Z_{G}$ computed on both trunk surfaces, with the MD interval in solid light gray, drawn on both sides around the first acquisition measurements. We notice no significant change for the $B S R$ and $Z_{G}$ profile; however, in the second acquisition, $\mathrm{X}_{\mathrm{G}}$ goes out of the $\mathrm{MD}$ interval in the thoracic region, indicating a real difference between acquisitions. This result is in agreement with the radiographic evaluation. At the fist visit, the Cobb angle was $41^{\circ}$ and $39^{\circ}$ in the thoracic and lumbar spine respectively, then after 8 months, there was a progression of $14^{\circ}$ and $5^{\circ}$ respectively.

\section{EVALUATION OF THE ESTHETIC OUTCOME: CASE STUDY}

The same procedure can also be used to assess the esthetic outcome after treatment. Figure 7 shows pre-operative and 6 months post-operative trunk surface acquisitions of an 11 years old female patient with scoliosis. Before surgery, the patient had a right thoracic curve of $68^{\circ}$ with apex at T10 and a left lumbar curve of $40^{\circ}$. After a posterior surgical approach and an anterior release of level $\mathrm{T} 7$ to $\mathrm{T} 11$, the thoracic curvature is about $9^{\circ}$ to the right indicating a good correction of the spinal alignment.

As illustrated in Figure 8, a significant difference in trunk surface pre- and post-operatively can be noticed in the three planes. First, in the axial plane, after surgery, there is less rotation of the back at the apex level (between T10 and T12); however we see an increasing negative rotation 
of the back between $\mathrm{C} 7 / \mathrm{T} 1$ and $\mathrm{T} 9$, suggesting the apparition of a rib hump in the upper left thoracic region of the back, after surgery. This particular finding could not have been noticed if we were analyzing the measurements only at the apex of the curve or at the most deformed level. In the coronal plane $\left(X_{G}\right.$ measurement), the trunk is less deviated to the right after surgery, even slightly deviated to the left suggesting an overcorrection of the spine frontal alignment. Indeed, in figure 7 , we see that before surgery, the waist is shifted to the right with respect to the pelvis, and after surgery, the waist is shifted to the left. Finally, in the sagittal plane $\left(Z_{G}\right.$ measurement), it is interesting to note that the trunk after surgery is tilted backward compared to the pre-operative trunk reconstruction.

\section{RELATIONSHIP WITH SPINAL DEFORMITY: CASE STUDY}

Even though the relationship between trunk and spinal measurements is beyond the scope of this paper, we present in Figure 9 a comparison between the functional BSR and the vertebrae axial rotation (VAR), the lateral deviation of the trunk $\left(\mathrm{X}_{\mathrm{G}}\right)$ and the lateral deviation of the vertebrae centroid $\left(\mathrm{X}_{\mathrm{V}}\right)$, and the posterior-anterior $(\mathrm{PA})$ deviation of the trunk $\left(\mathrm{Z}_{\mathrm{G}}\right)$ and the PA deviation of the vertebrae centroid $\left(Z_{V}\right)$, obtained for a 15 years old AIS female patient. The spinal measurements are computed from a $3 D$ reconstruction of the spine from lateral and posterior-anterior radiographs.

For the axial rotations, there seems to be a good correlation between BSR and VAR at all trunk levels. For the deviation in the coronal plane, $X_{G}$ and $X_{V}$ also show a good correlation, however, the amplitude of the deviations is smaller for the trunk. This has already been noted in the literature $[13,39,40]$ and may be explained by the soft tissues and the rib cage that try to attenuate the propagation of the deformity from the spine to the trunk surface. Finally, in the sagittal plane, we notice a good correlation between $Z_{G}$ and $Z_{V}$ only in the lumbar region. In the thoracic region, $Z_{G}$ is sensible to the anterior chest and particularly to the presence of breasts.

\section{Discussion}


To our knowledge, this is the first study to propose functional measurements for the analysis of trunk deformity in AIS. This new representation allows a full assessment that covers the entire trunk with high resolution. As previously mentioned by Scutt et al. [20], "measuring and evaluating only the 3D deformity and hump at the apex of a curve is clearly too simplistic". Taking the BSR in the clinical evaluation of the esthetic outcome (Figure 8) as an example, the maximum value along the trunk overlooks the extent of the hump above and below the apex of the curve.

The use of optical systems overcomes the limitations of multi-segmental scoliometer measurements $[20,21]$ since the acquisition is quasi-instantaneous and the patient is analyzed in the natural standing position. Furthermore, the resulting 3D model is of high resolution, making it possible to compute measurements over the whole length of the trunk. Several groups have previously proposed measurements computed on multiple trunk sections $[12,13,18]$. However, to facilitate analysis, they considered only the maximum values along the trunk. As proposed in this paper, the functional representation of the measurements makes it possible to consider all trunk levels in the analysis, using only 10 coefficients to fully describe each profile.

The new functional representation allowed us to evaluate the reliability of trunk surface measurements all over the trunk. As expected, the reliability is not the same at all trunk levels. Our measurements proved to be reliable below the shoulders level, thus, they are perfectly suited for the analysis of curves in the lumbar, thoraco-lumbar and main thoracic (below T3/T4) spine. The moderate reliability in the proximal thoracic spine (above T3/T4) is attributable to an imperfect trimming of the arms during the surface reconstruction process, resulting in asymmetric trunk sections at the shoulders level. In fact, the arms' trimming is operatordependent since this task is done manually prior to the reconstruction. In order to improve the analysis of proximal thoracic curves, future work will be oriented on a more standardized and automatic trimming of the arms. Moreover, the typical errors, particularly for the deviation measures, increase while going upward toward the VP. Additional experiments confirmed that 
this increase is mainly due to our patient-specific coordinate system's definition that do not compensate for upper trunk postural changes.

Even if measurements errors are reduced when considering the ( $\left.X^{\prime}, Y^{\prime}, Z^{\prime}\right)$ reference frame, the meaning of some measurements is modified. In fact, in the ( $\left.X^{\prime}, Y^{\prime}, Z^{\prime}\right)$ coordinate system, $X_{G}$ and $Z_{G}$ are equivalent to the $S R S$ vertebra deviation [31]. But in the $(X, Y, Z)$ coordinate system, they characterize respectively the trunk deviation in the coronal and sagittal plane, equivalent to the SRS global offset of the spine [31]. The latter is classically assessed during the physical examination, using a plumb line dropped from C7 vertebra [41]. The correlation analysis has shown a higher redundancy between the trunk deviation and the axial rotational components, whereas the trunk balance and the axial rotation together bring complementary information about the trunk deformity. This paper showed that, even if it appears less popular today, the idea of the plumb line test is pertinent in the clinical examination of AIS since it supplements the traditional scoliometer measurement. These measures refer to distinct phenomena of the same pathology [42]. However, the traditional plumb line test presents some limitations. Even if the C7 prominence is perfectly aligned with the middle of the buttocks, the trunk levels in between can be laterally deviated. The $X_{G}$ functional measurement overcomes this drawback since the trunk balance is assessed at all levels.

In the axial plane, BSR and TR are very strongly correlated. Both quantify axial rotational component. The tiny residual difference between those measurements is that the BSR encompasses the axial rotation of the trunk as well as the rib cage or lumbar muscles asymmetry. In future studies, where a compact set of measurements is required, one can easily choose only one of these two indices without losing much information.

With the results of the reliability study at hand, we computed the difference needed between separate measurements on a patient for the difference to be considered real. As presented in a case study, the proposed measurements were able to detect a significant progression of the spinal curvature. This constitutes a promising finding and opens the way to a less ionizing 
follow-up of AIS patients. A prospective study, following up a cohort of patients with mild AIS, is in progress to confirm this result and may help identifying eventual patterns of AIS progression. We also demonstrated that the proposed measurements can be used to assess the esthetic outcome of a surgical treatment. This can complement the radiographic outcome and document possible rib hump reassertion.

Currently, there exists no gold standard measure for assessing scoliosis trunk deformity. This is why previous work on trunk or back shape in this field tried to correlate topographic measurements to radiographic indices $[3,5]$, even though they refer to different aspects of scoliosis deformity. We demonstrated in a case study, that, although the range of the measurements is not the same, our trunk measurements profiles show similar variations to the spinal ones.

In conclusion, we proposed in this paper, new multi-level measurements for the non-invasive assessment of trunk surface deformities associated to scoliosis. The functional representation of the measurements allowed us to study their reliability not only at the most deformed level but at all trunk levels. The measurements proved to be reliable. We also identified, for all trunk sections, the minimal difference needed between acquisitions to consider a change as being real and not related to a change in the patient's positioning. As illustrated in the case studies, this confidence interval can be used to detect non-invasively a progression during scoliosis monitoring as well as to document the esthetic outcome of a surgery. 


\section{REFERENCES}

[1] Tones M, Moss N, Polly DW, Jr. A review of quality of life and psychosocial issues in scoliosis. Spine 2006;31:3027-38.

[2] Asher M, Lai SM, Burton D, et al. The influence of spine and trunk deformity on preoperative idiopathic scoliosis patients' health-related quality of life questionnaire responses. Spine 2004;29:861-8.

[3] Stokes IA, Moreland MS. Concordance of back surface asymmetry and spine shape in idiopathic scoliosis. Spine 1989;14:73-8.

[4] Liu XC, Thometz JG, Lyon RM, et al. Effects of trunk position on back surface-contour measured by raster stereophotography. Am J Orthop 2002;31:402-6.

[5] Goldberg CJ, Kaliszer M, Moore DP, et al. Surface topography, Cobb angles, and cosmetic change in scoliosis. Spine 2001;26:E55-63.

[6] Weisz I, Jefferson RJ, Turner-Smith AR, et al. ISIS scanning: a useful assessment technique in the management of scoliosis. Spine 1988;13:405-8.

[7] Poncet P, Delorme S, Ronsky JL, et al. Reconstruction of laser-scanned 3D torso topography and stereoradiographical spine and rib-cage geometry in scoliosis. Comput Methods Biomech Biomed Engin 2000;4:59-75.

[8] Dawson EG, Kropf MA, Purcell G, et al. Optoelectronic evaluation of trunk deformity in scoliosis. Spine 1993;18:326-31.

[9] Pazos V, Cheriet F, Song L, et al. Accuracy assessment of human trunk surface 3D reconstructions from an optical digitising system. Med Biol Eng Comput 2005;43:11-5.

[10] Turner-Smith AR, Harris JD, Houghton GR, et al. A method for analysis of back shape in scoliosis. J Biomech 1988;21:497-509.

[11] Berryman F, Pynsent P, Fairbank J, et al. A new system for measuring three-dimensional back shape in scoliosis. Eur Spine J 2008;17:663-72. 
[12] Stokes IA, Armstrong JG, Moreland MS. Spinal deformity and back surface asymmetry in idiopathic scoliosis. J Orthop Res 1988;6:129-37.

[13] Jaremko JL, Poncet P, Ronsky J, et al. Estimation of spinal deformity in scoliosis from torso surface cross sections. Spine 2001;26:1583-91.

[14] Suzuki N, Inami K, Ono T, et al. Analysis of Posterior Trunk Symmtery Index (POTSI) in scoliosis. Part 1. Stud Health Technol Inform 1999;59:81-4.

[15] Theologis TN, Jefferson RJ, Simpson AH, et al. Quantifying the cosmetic defect of adolescent idiopathic scoliosis. Spine 1993;18:909-12.

[16] Raso VJ, Lou E, Hill DL, et al. Trunk distortion in adolescent idiopathic scoliosis. J Pediatr Orthop 1998;18:222-6.

[17] Minguez MF, Buendia M, Cibrian RM, et al. Quantifier variables of the back surface deformity obtained with a noninvasive structured light method: evaluation of their usefulness in idiopathic scoliosis diagnosis. Eur Spine J 2007;16:73-82.

[18] Pazos V, Cheriet F, Danserau J, et al. Reliability of trunk shape measurements based on 3-D surface reconstructions. Eur Spine J 2007;16:1882-91.

[19] Burwell RG, Aujla RK, Cole AA, et al. Back shape assessment in each of three positions in preoperative patients with adolescent idiopathic scoliosis: evaluation of a 10-level Scoliometer method interpolated to 18-levels. Stud Health Technol Inform 2002;91:11922.

[20] Scutt ND, Dangerfield PH, Dorgan JC. The relationship between surface and radiological deformity in adolescent idiopathic scoliosis: effect of change in body position. Eur Spine $J$ 1996;5:85-90.

[21] Dangerfield PH, Williams L, Dorgan JC, et al. Multiple spinal level measurement of bodysurface deformity in scoliosis. In Alberti A, Drerup B, Hierholzer E eds. Surface Topography and Spinal Deformity VI, 1992:212-6. 
[22] Pratt RK, Webb JK, Burwell RG, et al. Changes in surface and radiographic deformity after Universal Spine System for right thoracic adolescent idiopathic scoliosis: is rib-hump reassertion a mechanical problem of the thoracic cage rather than an effect of relative anterior spinal overgrowth? Spine 2001;26:1778-87.

[23] Murrell GA, Coonrad RW, Moorman CT, 3rd, et al. An assessment of the reliability of the Scoliometer. Spine 1993;18:709-12.

[24] Amendt LE, Ause-Ellias KL, Eybers JL, et al. Validity and reliability testing of the Scoliometer. Phys Ther 1990;70:108-17.

[25] Ramsay JO, Dalzell CJ. Some tools for functional data-analysis. Journal of the Royal Statistical Society Series B-Methodological 1991;53:539-72.

[26] Song JJ, Lee HJ, Morris JS, et al. Clustering of time-course gene expression data using functional data analysis. Computational Biology and Chemistry 2007;31:265-74.

[27] Park C, Koo JY, Kim S, et al. Classification of gene functions using support vector machine for time-course gene expression data. Computational Statistics \& Data Analysis 2008;52:2578-87.

[28] Duhamel A, Devos P, Bourriez JL, et al. Functional data analysis for gait curves study in Parkinson's disease. In Hasman A, Haux R, VanderLei J, et al. eds. Ubiquity: Technologies for Better Health in Aging Societies, 2006:569-74.

[29] Roislien J, Skare O, Gustavsen M, et al. Simultaneous estimation of effects of gender, age and walking speed on kinematic gait data. Gait \& Posture 2009;30:441-5.

[30] Donoghue OA, Harrison AJ, Coffey $\mathrm{N}$, et al. Functional data analysis of running kinematics in chronic Achilles tendon injury. Medicine and Science in Sports and Exercise 2008;40:1323-35.

[31] Stokes IA. Three-dimensional terminology of spinal deformity. A report presented to the Scoliosis Research Society by the Scoliosis Research Society Working Group on 3-D terminology of spinal deformity. Spine 1994;19:236-48. 
[32] Fitzgibbon A, Pilu M, Fisher RB. Direct least square fitting of ellipses. IEEE Transactions on Pattern Analysis and Machine Intelligence 1999;21:476-80.

[33] Ramsay JO, Silverman BW. Functional data analysis. Ed. New-York: Springer, 2002.

[34] Seoud L, Dansereau J, Labelle H, Cheriet F. A Novel Method for Non Invasive Clinical Assessment of Scoliosis from Trunk Shape Analysis. Medical Image Analysis. Submitted in Nov. 2011.

[35] Shrout PE, Fleiss JL. Intraclass correlations: uses in assessing rater reliability. Psychol Bull. 1979;86:420-8.

[36] Weir JP. Quantifying test-retest reliability using the intraclass correlation coefficient and the SEM. Journal of Strength and Conditioning Research 2005;19:231-40.

[37] Hopkins WG. Measures of reliability in sports medicine and science. Sports Medicine 2000;30:1-15.

[38] I. A. Stokes, J. G. Armstrong, and M. S. Moreland, "Spinal deformity and back surface asymmetry in idiopathic scoliosis," J Orthop Res, vol. 6, pp. 129-37, 1988.

[39] I. A. Stokes, "Axial rotation component of thoracic scoliosis," J Orthop Res, vol. 7, pp. 7028, 1989.

[40] Stagnara P. Spinal deformity. London: Butterworths; 1988.

[41] Grosso C, Negrini S, Boniolo A, Negrini AA. The validity of clinical examination in adolescent spinal deformities. Stud Health Technol Inform 2002; 91: 123-125. 


\section{FIGURE LEGENDS}

Figure 1 - Graphical interface for trunk measurements visualization. A 3D view of the trunk is displayed on the bottom left of the interface. The functional measurements are plotted on the upper right. Using the slider, the user can choose a trunk level to display on the top left.

Figure 2 - Root mean squared error (RMSE) between raw data and functional data as a function of the number $(K)$ of cubic B-spline basis used for smoothing the functional data.

Figure 3 - Test-retest reliability for $f_{B S R}, f_{T R}, f_{X G}, f_{Z G}$ computed in the $(X, Y, Z)$ coordinate system. The intra-class correlation coefficients (on the left) and the typical errors of measurement (on the right) are plotted as functional data along the trunk, for each measurement.

Figure 4 - Typical errors of measurement for $f_{B S R}, f_{T R}, f_{X G}, f_{Z G}$, computed in the (X', $\left.Y^{\prime}, Z^{\prime}\right)$ coordinate system plotted as functional data along the trunk, for each measurement.

Figure 5 - Trunk surface acquisitions of a 16 years old male AIS patient, taken at 8 months interval.

Figure 6 - Comparison between functional BSR, $X_{G}$ and $Z_{G}$ computed on the first acquisition (dashed black lines) and on the second acquisition (solid red lines) taken 8 months later of a same 16 years old male AIS patient. The light gray intervals correspond to the minimal difference needed between the two observations for the difference to be considered real. As indicated by the black arrow, after 8 months follow-up, the trunk is significantly more deviated to the right in the mid-thoracic region. No real change is observed in the axial and sagittal planes.

Figure 7 - Trunk surface acquisitions of an 11 years old female patient with scoliosis, taken before and 6 months after spinal surgery.

Figure 8 - Comparison between functional $B S R, X_{G}$ and $Z_{G}$ computed on the first acquisition (dashed black lines) and on the second acquisition (solid red lines) 6 months after spinal surgery of an 11 years old female patient with scoliosis. The light gray intervals correspond to 
the minimal difference needed between the two observations for the difference to be considered real. Significant changes are observed for the three measurements.

Figure 9 - Relationship between spinal measurements (red dots) computed on 3D reconstructions of the spine and functional trunk measurements computed on trunk surface reconstruction (solid blue lines) of a 15 years old female AIS patients.

\section{Figure 1}

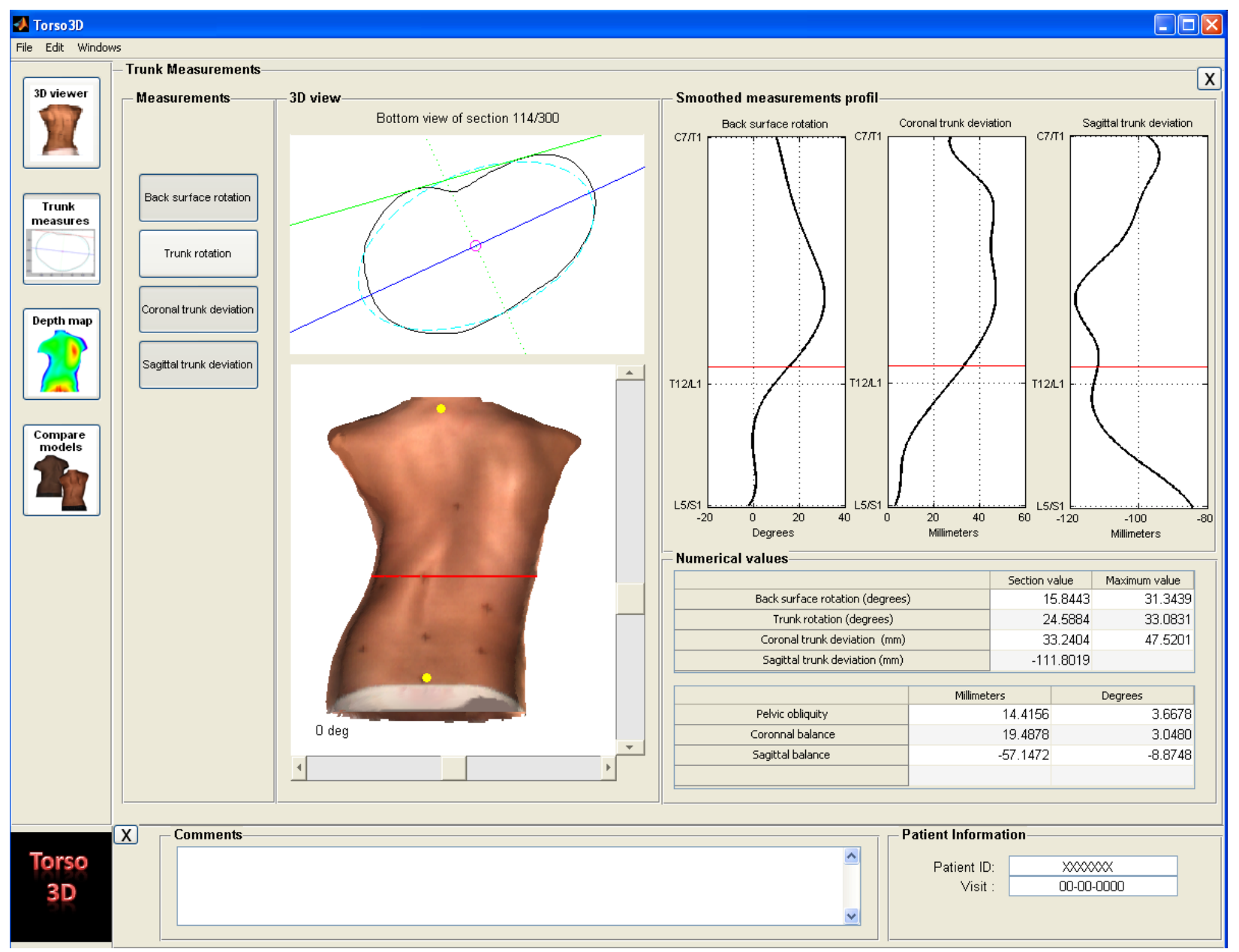


Figure 2

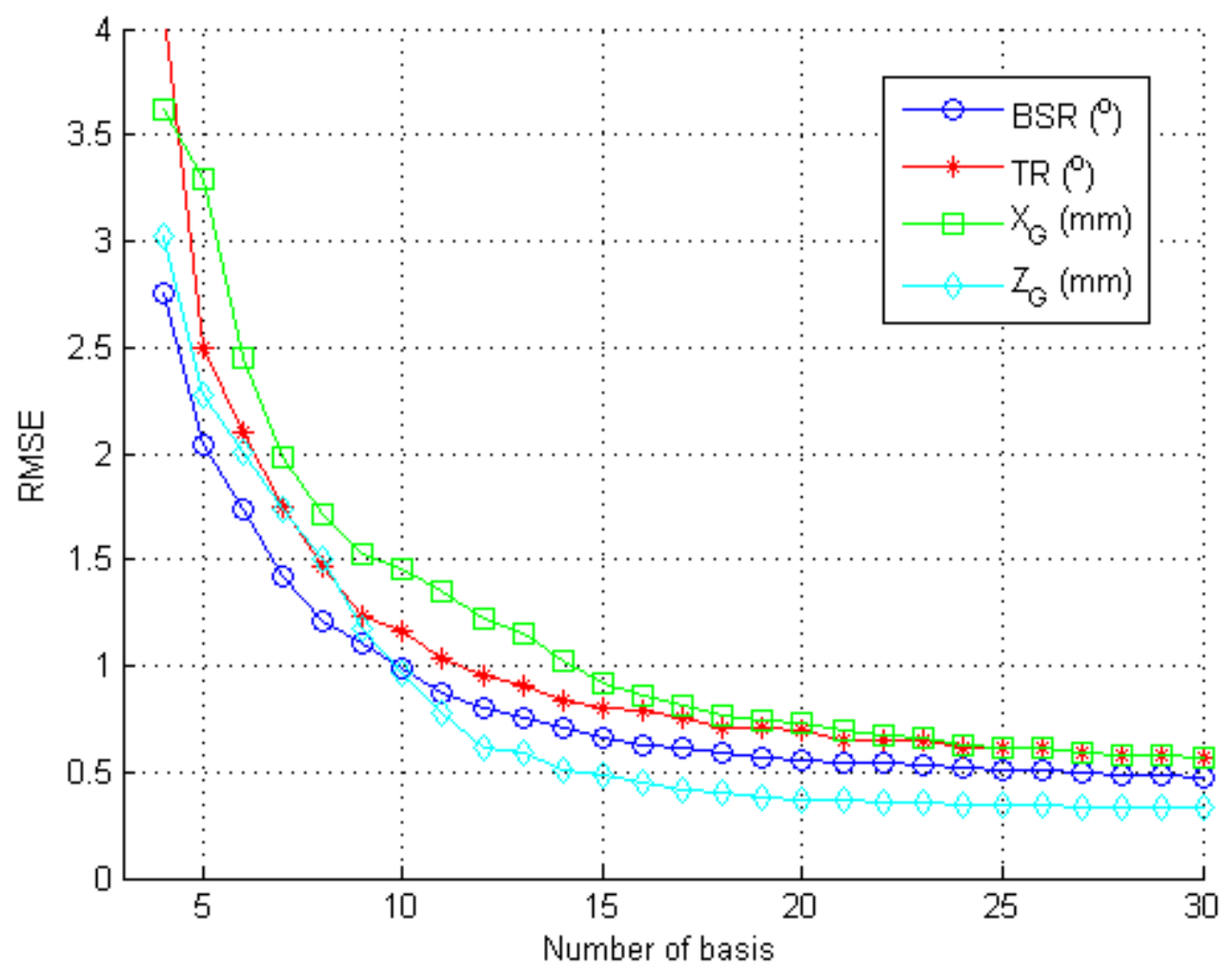

Figure 3
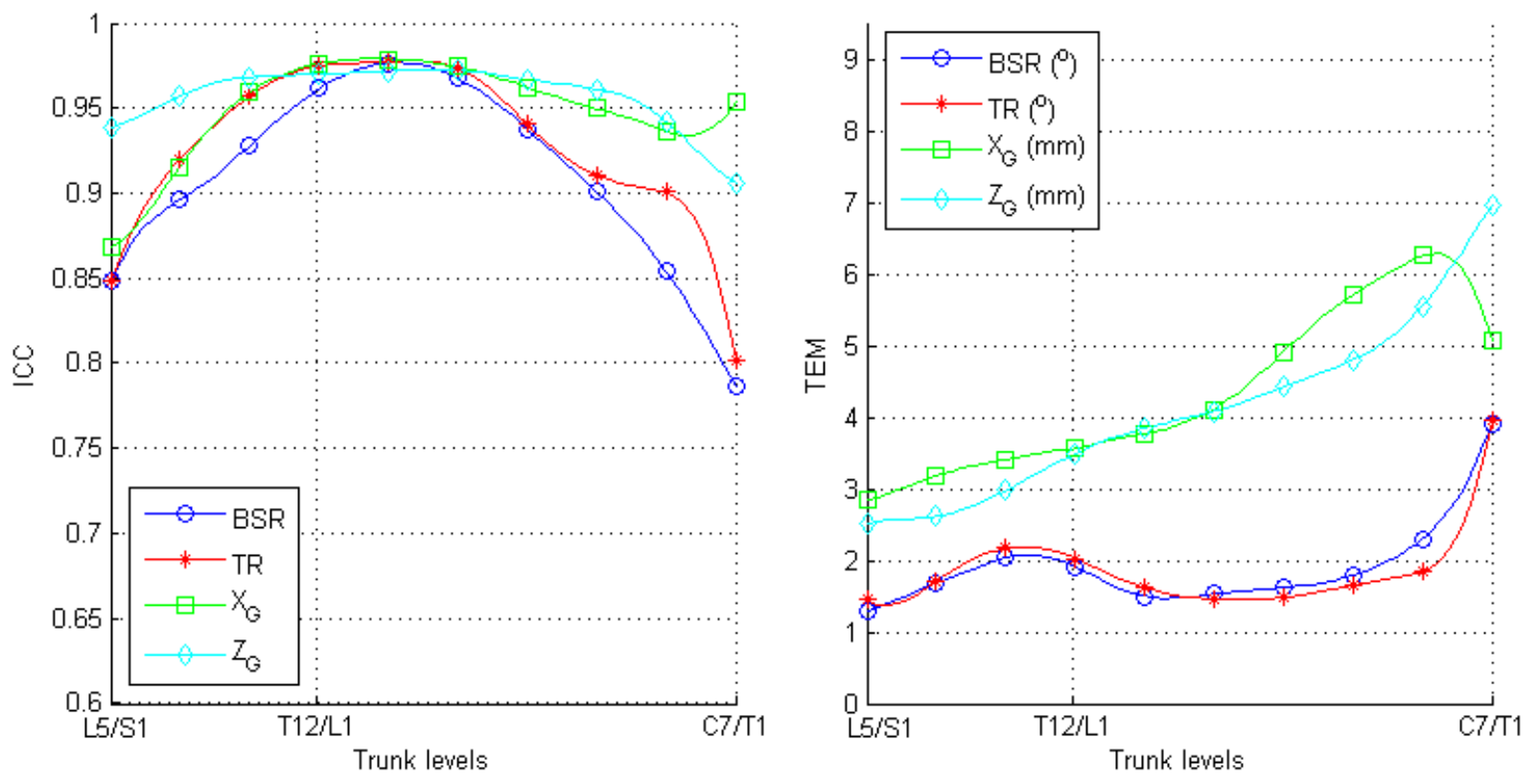
Figure 4

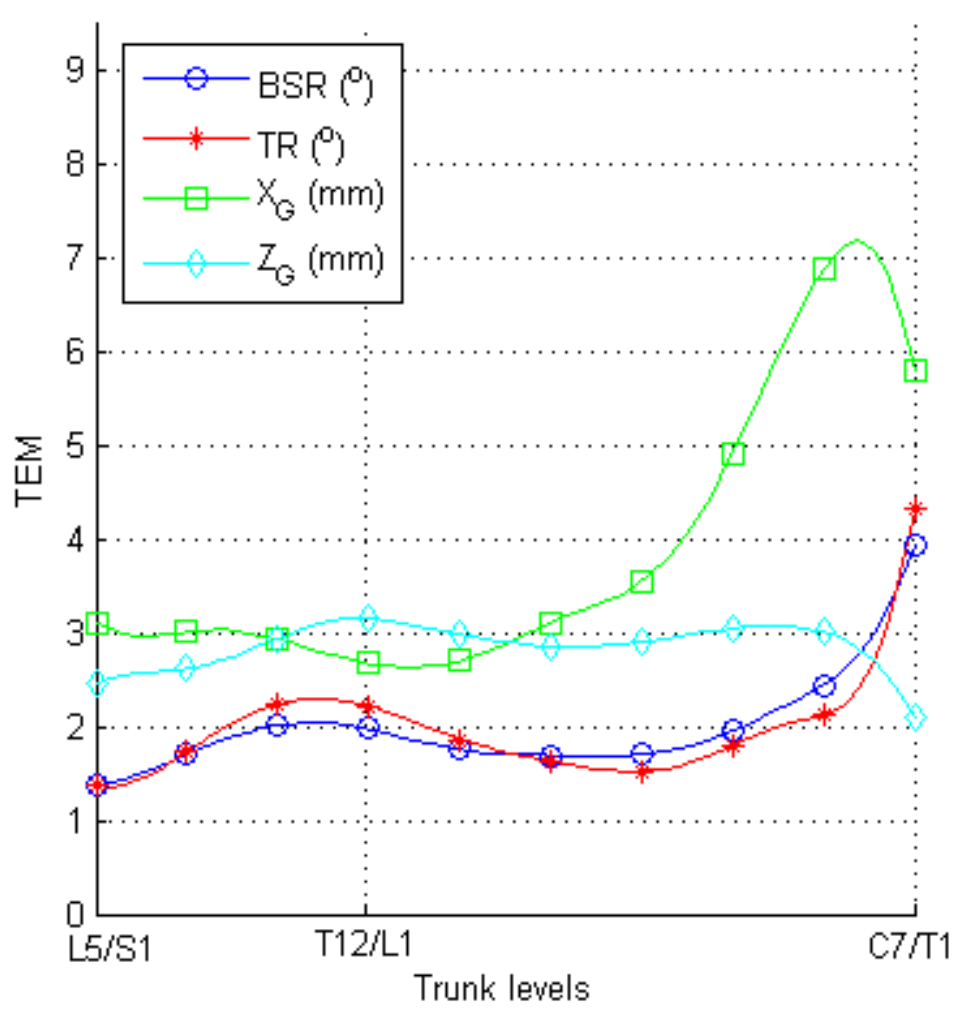

Figure 5

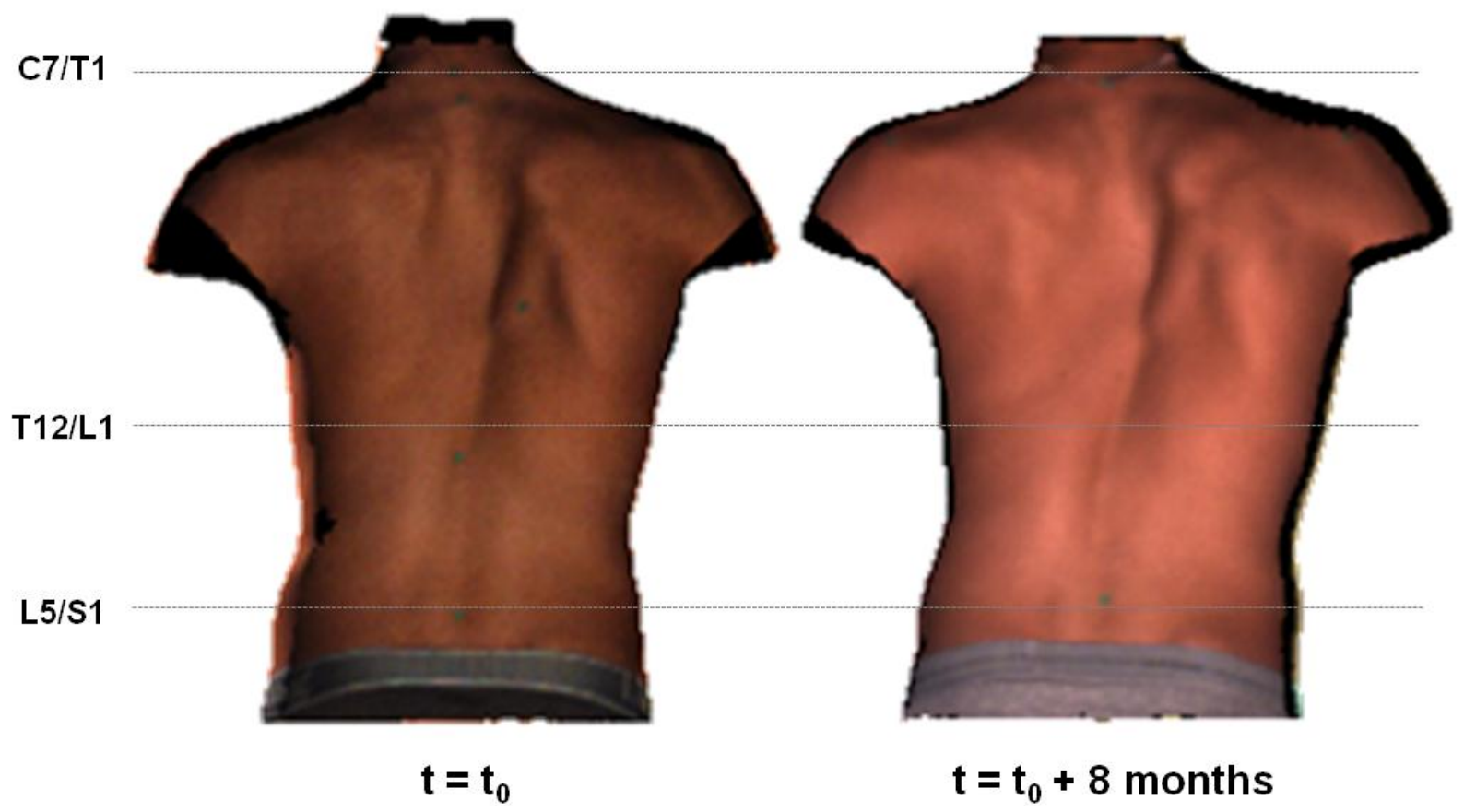


Figure 6
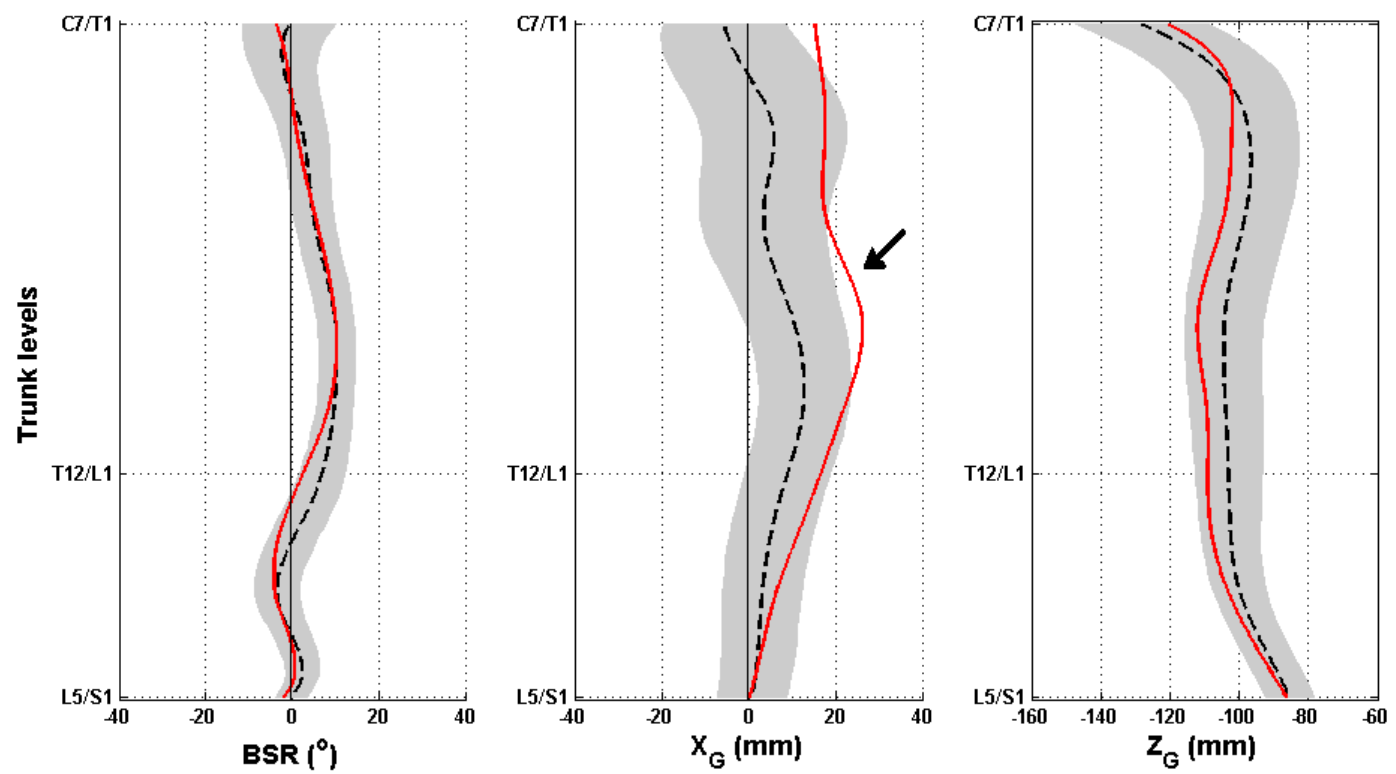

Figure 7

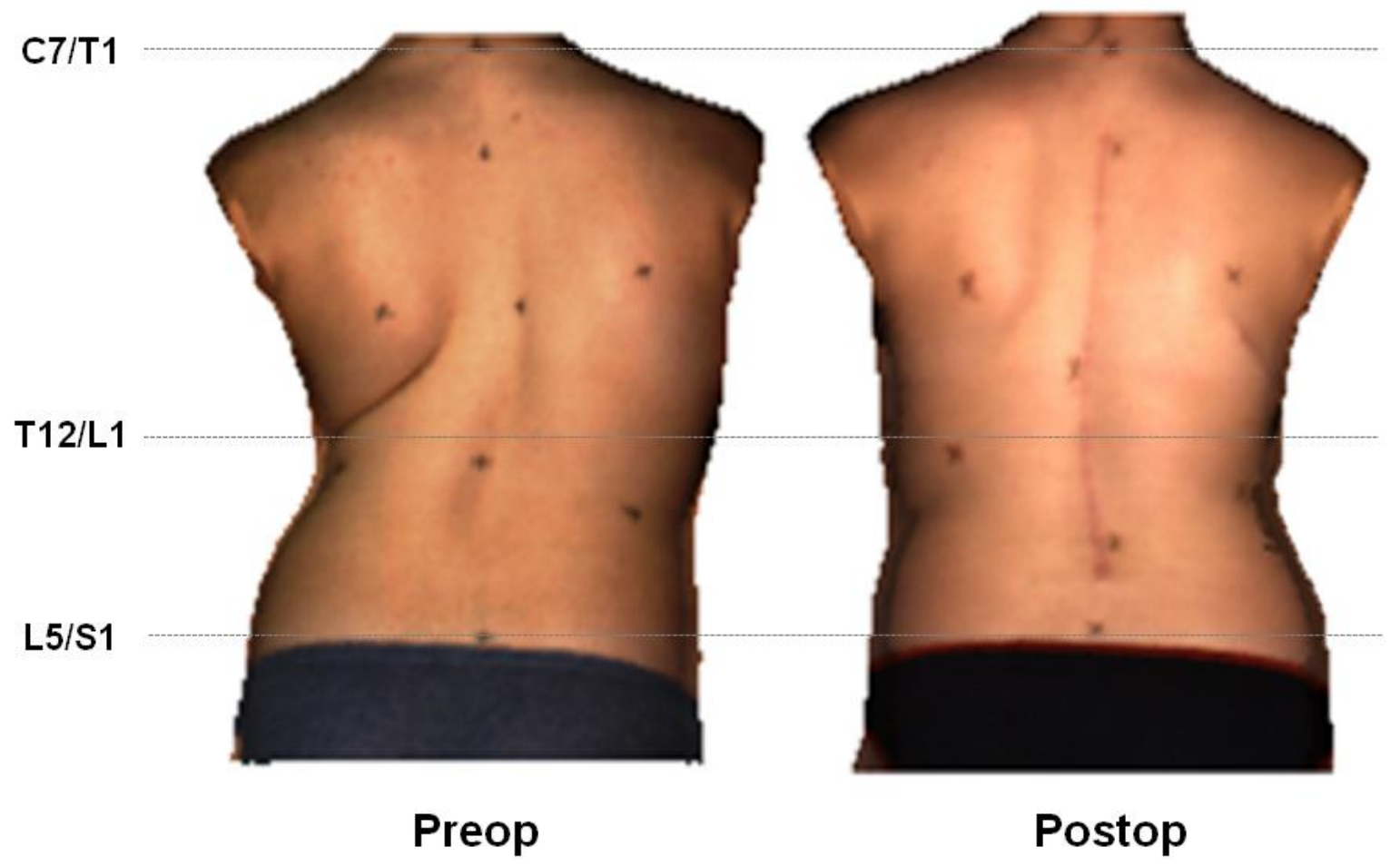


Figure 8
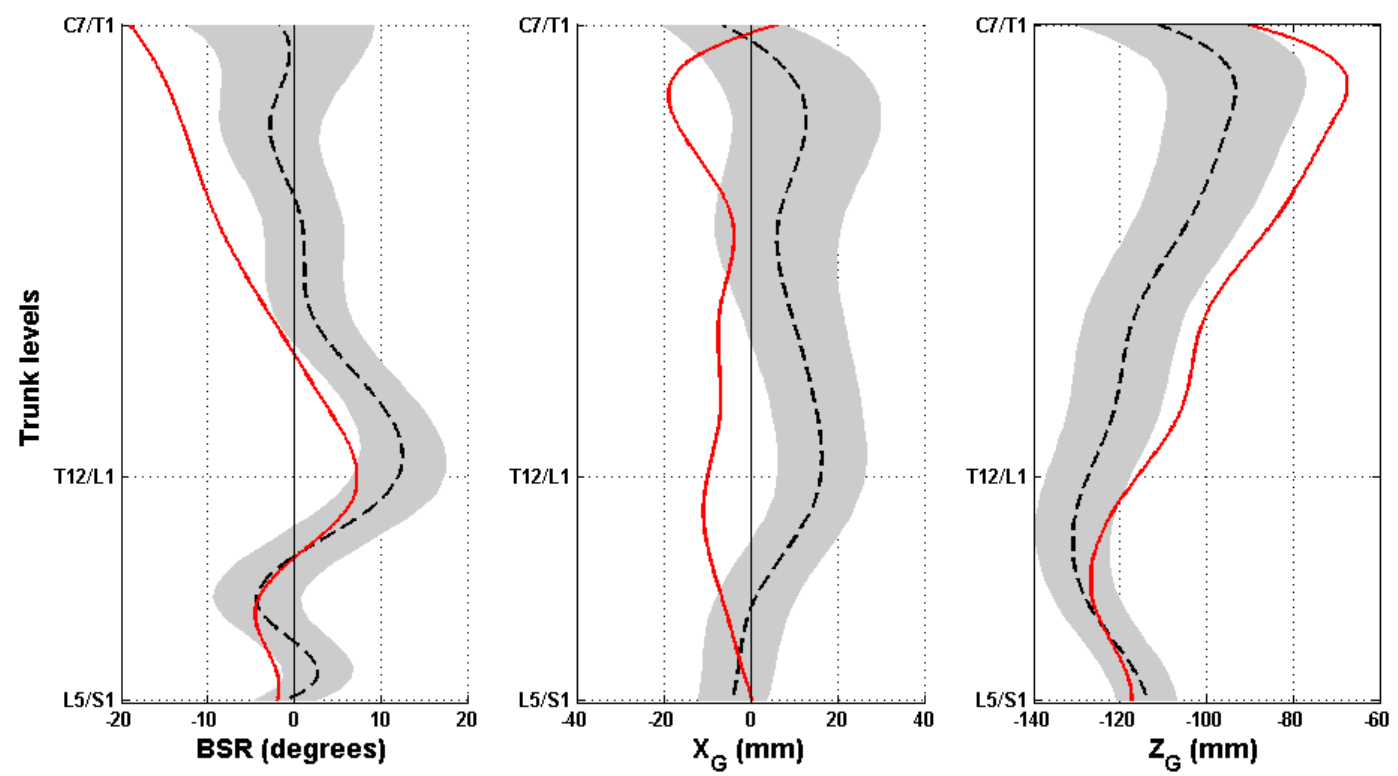

Figure 9
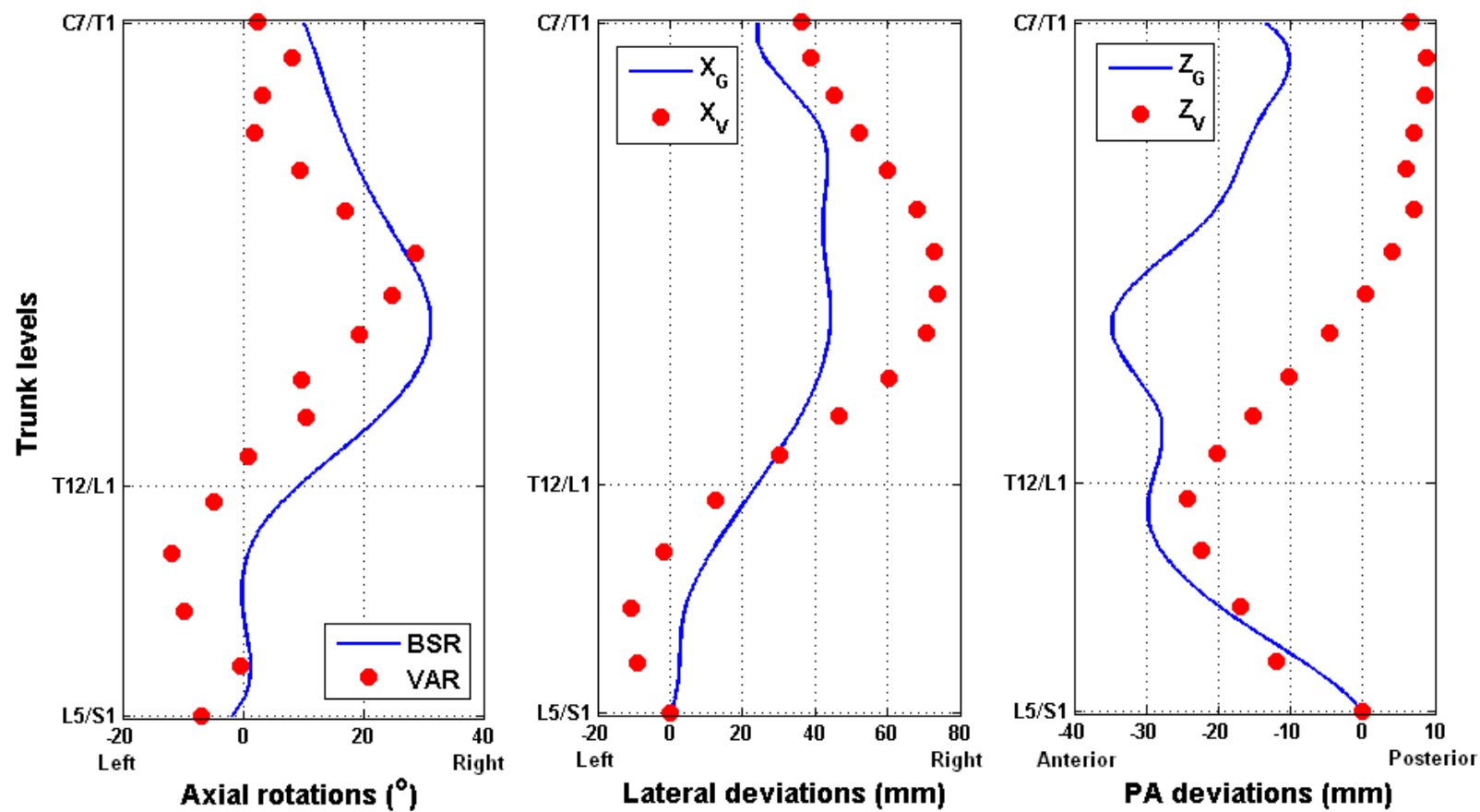


\section{TABLES}

Table 1 - Information about the cohort

\begin{tabular}{ccccc}
\hline $\mathbf{N}=32$ & $\begin{array}{c}\text { Major Cobb } \\
\text { angle }\left(^{\circ}\right)\end{array}$ & $\begin{array}{c}\text { Age } \\
(\text { year) }\end{array}$ & $\begin{array}{c}\text { Weight } \\
(\mathbf{k g})\end{array}$ & $\begin{array}{c}\text { Height } \\
(\mathbf{c m})\end{array}$ \\
\hline $\begin{array}{c}\text { Mean } \\
\begin{array}{c}\text { Standard } \\
\text { deviation }\end{array}\end{array}$ & 54.3 & 14.3 & 47.5 & 159.4 \\
Range & 15.3 & 1.7 & 9.6 & 8.8 \\
\hline
\end{tabular}

$\mathrm{N}$ : number of patients 